九州大学学術情報リポジトリ

Kyushu University Institutional Repository

\title{
AN EDGEWORTH EXPANSION AND A NORMALIZING TRANSFORMATION FOR L-STATISTICS
}

Maesono, Yoshihiko

Faculty of Mathematics, Kyushu University

https://doi.org/10.5109/16772

出版情報: Bulletin of informatics and cybernetics. 39, pp. 25-43，2007-12. Research Association of Statistical Sciences

バージョン :

権利関係 : 


\section{AN EDGEWORTH EXPANSION AND A NORMALIZING TRANSFORMATION FOR $L$-STATISTICS}

by

Yoshihiko MAESONo

Reprinted from the Bulletin of Informatics and Cybernetics

Research Association of Statistical Sciences, Vol.39

FUKUOKA, JAPAN

2007 


\title{
AN EDGEWORTH EXPANSION AND A NORMALIZING TRANSFORMATION FOR $L$-STATISTICS
}

\author{
By
}

\author{
Yoshihiko MAESONo*
}

\begin{abstract}
We obtain a stochastic approximation of a jackknife variance estimator of an $L$-statistic and also derive an approximation of a studentized $L$-statistic, in which we substitute the jackknife variance estimator. An Edgeworth expansion with remainder term $o\left(n^{-1 / 2}\right)$ is established for the studentized $L$-statistic. Using the Edgeworth expansion, we also discuss a normalizing transformation which improves the accuracy of the coverage probability of confidence interval.
\end{abstract}

Key Words and Phrases: Edgeworth expansion, Gini's mean difference, $H$-decomposition, Jackknife estimator of variance, Normalizing transformation, Studentized $L$-statistics.

\section{Introduction}

Let $X_{1}, \cdots, X_{n}$ be independently and identically distributed random variables with distribution function $F(x)$. Let $J(u)$ be a score function and $F_{n}(u)$ be an empirical distribution function, i.e.

$$
F_{n}(u)=n^{-1} \sum_{i=1}^{n} I\left(X_{i} \leq u\right)
$$

where $I(\cdot)$ is an indicator function. Then an $L$-statistic is given by

$$
T\left(F_{n}\right)=\int_{0}^{1} F_{n}^{-1}(u) J(u) d u
$$

where $F_{n}^{-1}(u)=\inf \left\{x ; F_{n}(x) \geq u\right\} . T\left(F_{n}\right)$ is regarded as an estimator of $\mathrm{T}(\mathrm{F})$

$$
T(F)=\int_{0}^{1} F^{-1}(u) J(u) d u,
$$

and $T\left(F_{n}\right)$ constitutes a subclass of $L$-statistics (see Serfling (1980)). The asymptotic variance of $\sqrt{n}\left(T\left(F_{n}\right)-T(F)\right)$ is given by

$$
\sigma^{2}(J, F)=\int_{-\infty}^{\infty} \int_{-\infty}^{\infty} J(F(u)) J(F(t))[F(\min (u, t))-F(u) F(t)] d u d t .
$$

For a standardized $L$-statistic $\left.\sqrt{n}\left\{T\left(F_{n}\right)\right)-T(F)\right\} / \sigma(J, F)$, Helmers (1982) obtained an Edgeworth expansion with remainder term $o\left(n^{-1 / 2}\right)$ and Alberink, Pap and van Zuijlen (2001) discussed Edgeworth expansion of the standardized $L$-statistic under weaker conditions of $J(u)$.

\footnotetext{
* Faculty of Mathematics, Kyushu University, Japan. e-mail address: maesono@ math.kyushu-u.ac.jp
} 
A jackknife variance estimator of $\sqrt{n}\left(T\left(F_{n}\right)-T(F)\right)$ is given by

$$
\hat{\sigma}^{2}(J, F)=(n-1) \sum_{i=1}^{n}\left[T\left(F_{n ; i}\right)-T\left(F_{n}\right)\right]^{2}
$$

where $F_{n ; i}$ is an empirical distribution function based on a sample of $n-1$ points with $X_{i}$ left out. Parr and Schucany (1982) show that $\hat{\sigma}^{2}(J, F)$ is a consistent estimator of $\sigma^{2}(J, F)$ under some regularity conditions. Shao (1991) also showed the strong consistency of the jackknife variance estimator for a generalized $L$-statistics, which is proposed by Serfling (1984) and includes $T\left(F_{n}\right)$. In this paper we will obtain a stochastic approximation of the estimator $\hat{\sigma}^{2}(J, F)$ and study an Edgeworth expansion of the studentized $L$-statistic

$$
\frac{\sqrt{n}\left\{T\left(F_{n}\right)-T(F)\right\}}{\hat{\sigma}(J, F)} .
$$

Let us define

$$
K(u)=\int_{0}^{u} J(v) d v
$$

Boos and Serfling (1979) (see Serfling (1980) p.265) showed that

$$
T\left(F_{n}\right)-T(F)=-\int_{-\infty}^{\infty}\left[K\left(F_{n}(u)\right)-K(F(u))\right] d u .
$$

Using this form, we will discuss asymptotic properties of the jackknife variance estimator and the studentized $L$-statistic.

In Section 2, using the form (2) and the $H$-decomposition, which is due to Hoeffding (1961), we will obtain the stochastic approximation of the jackknife estimator $\hat{\sigma}^{2}(J, F)$ with remainder term $o_{L}\left(n^{-1 / 2}\right)$ where

$$
P\left\{\left|o_{L}\left(n^{-1 / 2}\right)\right| \geq c n^{-1 / 2}(\log n)^{-1}\right\}=o\left(n^{-1 / 2}\right)
$$

for some constant $c>0$. We will also obtain a stochastic approximation of the studentized $L$ statistic. In Section 3, we will discuss the Edgeworth expansion with remainder term $o\left(n^{-1 / 2}\right)$ and obtain a normalizing transformation that improves the convergence rate of a confidence interval for $T(F)$.

\section{Stochastic approximation of jackknife variance estimator and studentized $L$-statistic}

In the sequel we use one symbol $o_{L}\left(n^{-1 / 2}\right)$ which satisfies the equation (3). Note that $o_{L}\left(n^{-1 / 2}\right)+o_{L}\left(n^{-1 / 2}\right)$ and $o_{L}\left(n^{-1 / 2}\right) o_{L}\left(n^{-1 / 2}\right)$ are also $o_{L}\left(n^{-1 / 2}\right)$. From the Markov's inequality, if $E|R|^{\alpha}=O\left(n^{-1 / 2-\alpha / 2-\delta}\right)(\alpha>0, \delta>0)$, we have that $R=o_{L}\left(n^{-1 / 2}\right)$. We will obtain stochastic approximations of the jackknife variance estimator and the studentized $L$-statistic with remainder term $o_{L}\left(n^{-1 / 2}\right)$.

First we obtain the stochastic approximation of the jackknife variance estimator $\hat{\sigma}^{2}(J, F)$. Let us define

$$
k(u, t)=F(\min (u, t))-F(u) F(t)
$$


and

$$
\begin{aligned}
\alpha_{1}\left(X_{i}\right)=\int_{-\infty}^{\infty} \int_{-\infty}^{\infty}[ & J(F(u)) J(F(t))\left\{\tilde{I}\left(X_{i} ; u\right) \tilde{I}\left(X_{i} ; t\right)-k(u, t)\right\} \\
+ & \left.+2 J(F(u)) J^{(1)}(F(t)) k(u, t) \tilde{I}\left(X_{i} ; t\right)\right] d u d t
\end{aligned}
$$

where $\tilde{I}\left(X_{i} ; u\right)=I\left(X_{i} \leq u\right)-F(u)$. Then we have the following stochastic approximation of $\hat{\sigma}^{2}(J, F)$.

Theorem 1. Assume that $J^{(1)}(u)$ is bounded for $0 \leq u \leq 1$ and $J^{(2)}(u)$ satisfies a Lipshitz condition for order $s>0$, i.e. $\left|J^{(2)}(u)-J^{(2)}(v)\right| \leq H|u-v|^{s}$ for some $H>0$. If

$$
\int_{-\infty}^{\infty}\{F(u)(1-F(u))\}^{1 / 4}<\infty
$$

we have

$$
\hat{\sigma}^{2}(J, F)=\sigma^{2}(J, F)+n^{-1} \sum_{i=1}^{n} \alpha_{1}\left(X_{i}\right)+o_{L}\left(n^{-1 / 2}\right) .
$$

It is easy to see that $E\left[\alpha\left(X_{1}\right)\right]=0$. Using this stochastic approximation, we can discuss asymptotic properties of $\hat{\sigma}^{2}(J, F)$ and obtain the Edgeworth expansion of the studentized $L$ statistic.

Since the Edgeworth expansion has a bounded derivative, if $P\left\{|R| \geq n^{-1 / 2} \varepsilon_{n}\right\}=o\left(n^{-1 / 2}\right)$ for $\varepsilon_{n} \rightarrow 0$, we can ignore $R$ when we discuss asymptotic distribution with remainder term $o\left(n^{-1 / 2}\right)$. Using the stochastic approximation of the jackknife variance estimator $\hat{\sigma}^{2}(J, F)$, we will obtain an stochastic approximation of the studentized $L$-statistic $\sqrt{n}\left\{T\left(F_{n}\right)-T(F)\right\} /$ $\hat{\sigma}(J, F)$. Let us define

$$
\begin{aligned}
\eta & =-\frac{1}{2} \int_{-\infty}^{\infty} J^{(1)}(F(u)) F(u)(1-F(u)) d u, \\
g_{1}\left(X_{i}\right) & =-\int_{-\infty}^{\infty} J(F(u)) \tilde{I}\left(X_{i} ; u\right) d u
\end{aligned}
$$

and

$$
g_{2}\left(X_{i}, X_{j}\right)=-\int_{-\infty}^{\infty} J^{(1)}(F(u)) \tilde{I}\left(X_{i} ; u\right) \tilde{I}\left(X_{j} ; u\right) d u .
$$

Then we have the following theorem.

Theorem 2. Under the same conditions of Theorem 1, we have

$$
\begin{aligned}
& T\left(F_{n}\right)-T(F) \\
= & n^{-1} \eta+n^{-1} \sum_{i=1}^{n} g_{1}\left(X_{i}\right)+n^{-2} \sum_{C_{n, 2}} g_{2}\left(X_{i}, X_{j}\right)+n^{-1 / 2} o_{L}\left(n^{-1 / 2}\right)
\end{aligned}
$$

and

$$
\hat{\sigma}(J, F)=\sigma(J, F)+n^{-1} \sum_{i=1}^{n} \frac{\alpha_{1}\left(X_{i}\right)}{2 \sigma(J, F)}+o_{L}\left(n^{-1 / 2}\right) .
$$


Using these stochastic approximations, we can obtain a stochastic approximation of the studentized $L$-statistic. Let us define

$$
\begin{aligned}
\tau & =\frac{\eta}{\sigma(J, F)}-\frac{E\left[\alpha\left(X_{1}\right) g_{1}\left(X_{1}\right)\right]}{2 \sigma^{3}(J, F)}, \\
v_{1}(x) & =\frac{g_{1}(x)}{\sigma(J, F)}
\end{aligned}
$$

and

$$
v_{2}(x, y)=\frac{g_{2}(x, y)}{\sigma(J, F)}-\frac{1}{2 \sigma^{3}(J, F)}\left\{\alpha_{1}(x) g_{1}(y)+\alpha_{1}(y) g_{1}(x)\right\} .
$$

Thus we have the following theorem.

Theorem 3. Under the same conditions of Theorem 1, we have

$$
\begin{aligned}
& \frac{\sqrt{n}\left(T\left(F_{n}\right)-T(F)\right)}{\hat{\sigma}(J, F)} \\
= & n^{-1 / 2} \tau+n^{-1 / 2} \sum_{i=1}^{n} v_{1}\left(X_{i}\right)+n^{-3 / 2} \sum_{C_{n, 2}} v_{2}\left(X_{i}, X_{j}\right)+R_{n}
\end{aligned}
$$

where

$$
P\left\{\left|R_{n}\right| \geq n^{-1 / 2} \varepsilon_{n}\right\}=o\left(n^{-1 / 2}\right)
$$

for $\varepsilon_{n} \rightarrow 0$.

Theorem 3 shows that the studentized $L$-statistic is an asymptotic $U$-statistic. For the asymptotic $U$-statistic, Lai and Wang (1993) obtained an Edgeworth expansion. Using the stochastic approximation of Theorem 3, we can obtain the Edgeworth expansion of the studentized $L$-statistic.

\section{Edgeworth expansion and normalizing transformation}

Let us assume the following conditions.

$\left(C_{1}\right) \quad E\left\{\left|v_{1}\left(X_{1}\right)\right|^{4}+\left|v_{3}\left(X_{1}, X_{2}, X_{3}\right)\right|^{4}\right\}<\infty$ and $\sigma^{2}(J, F)>0$.

$\left(C_{2}\right) \quad \lim \sup _{|t| \rightarrow \infty} \mid E\left[\exp \left\{\right.\right.$ it $\left.\left.v_{1}\left(X_{1}\right)\right\}\right] \mid<1$.

Let us define

$$
\begin{aligned}
\kappa_{3} & =E\left[v_{1}^{3}\left(X_{1}\right)\right]+3 E\left[v_{1}\left(X_{1}\right) v_{1}\left(X_{2}\right) v_{2}\left(X_{1}, X_{2}\right)\right], \\
P_{1}(x) & =\frac{\kappa_{3}\left(x^{2}-1\right)}{6}
\end{aligned}
$$

and then it follows from Lai and Wang (1993) that

$$
\begin{aligned}
& P\left\{\frac{\sqrt{n}\left\{T\left(F_{n}\right)-T(F)\right\}}{\hat{\sigma}(J, F)}-n^{-1 / 2} \tau \leq x\right\} \\
= & \Phi(x)-n^{-1 / 2} \phi(x) P_{1}(x)+o\left(n^{-1 / 2}\right) .
\end{aligned}
$$


Thus expanding with respect to $n^{-1 / 2} \tau$, from the standard argument (see Petrov (1995) p.16), we have the Edgeworth expansion of $\sqrt{n}\left\{T\left(F_{n}\right)-T(F)\right\} / \hat{\sigma}(J, F)$ as follows.

Theorem 4. If the conditions of Theorem 1 and $C_{1}, C_{2}$ hold, we have

$$
\sup _{x}\left|P\left\{\frac{\sqrt{n}\left\{T\left(F_{n}\right)-T(F)\right\}}{\hat{\sigma}(J, F)} \leq x\right\}-Q_{n}(x)\right|=o\left(n^{-1 / 2}\right) .
$$

where

$$
Q_{n}(x)=\Phi(x)-n^{-1 / 2} \phi(x)\left\{P_{1}(x)+\tau\right\} .
$$

As pointed out by many authors, we cannot improve the convergence rates by inverting the above Edgeworth expansion. Konishi (1981) and Hall (1992) proposed normalizing transformations which remove bias and skewness. Here we consider the transformation $\pi(\cdot)$ which satisfies

$$
\sup _{x}\left|P\left\{\pi\left(\frac{\sqrt{n}\left\{T\left(F_{n}\right)-T(F)\right\}}{\hat{\sigma}(J, F)}\right) \leq x\right\}-\Phi(x)\right|=o\left(n^{-1 / 2}\right) .
$$

Using this transformation, we can construct a confidence interval of $T(F)$, which improve the convergence rate.

Fujioka and Maesono (2000) have obtained the normalizing transformation for asymptotic $U$-statistics. Applying their result, we can get the transformation which satisfies the equation (6). Let us define

$$
\begin{aligned}
p & =-\frac{1}{6} E\left[v_{1}^{3}\left(X_{1}\right)\right]-\frac{1}{2} E\left[v_{1}\left(X_{1}\right) v_{1}\left(X_{2}\right) v_{2}\left(X_{1}, X_{2}\right)\right] \\
q & =\frac{1}{6} E\left[v_{1}^{3}\left(X_{1}\right)\right]+\frac{1}{2} E\left[v_{1}\left(X_{1}\right) v_{1}\left(X_{2}\right) v_{2}\left(X_{1}, X_{2}\right)\right]-\tau,
\end{aligned}
$$

and $\hat{p}$ and $\hat{q}$ are consistent estimators of $p$ and $q$, which satisfy

$$
n^{-1 / 2} \hat{p}=n^{-1 / 2} p+o_{L}\left(n^{-1 / 2}\right) \quad \text { and } \quad n^{-1 / 2} \hat{q}=n^{-1 / 2} q+o_{L}\left(n^{-1 / 2}\right) .
$$

Define

$$
\pi(s)=s+\frac{\hat{p}}{\sqrt{n}} s^{2}+\frac{\hat{q}}{\sqrt{n}}+\frac{\hat{p}^{2}}{3 n} s^{3} .
$$

Then, from Fujioka and Maesono (2000), we have the following theorem.

Theorem 5. If the conditions of Theorem 4 and the equation (7) hold, we have

$$
\sup _{x}\left|P\left\{\pi\left(\frac{\sqrt{n}\left\{T\left(F_{n}\right)-T(F)\right\}}{\hat{\sigma}(J, F)}\right) \leq x\right\}-\Phi(x)\right|=o\left(n^{-1 / 2}\right) .
$$

Let us define

$$
\begin{aligned}
& m\left(u_{1}, u_{2}, u_{3}\right)=F\left(\min \left(u_{1}, u_{2}, u_{3}\right)\right)- F\left(u_{1}\right) F\left(\min \left(u_{2}, u_{3}\right)\right) \\
&-F\left(u_{2}\right) F\left(\min \left(u_{1}, u_{3}\right)\right)-F\left(u_{3}\right) F(\left.\min \left(u_{1}, u_{2}\right)\right)+F\left(u_{1}\right) F\left(u_{2}\right) F\left(u_{3}\right), \\
& e_{1}=-\int_{-\infty}^{\infty} \int_{-\infty}^{\infty} \int_{-\infty}^{\infty} J\left(F\left(u_{1}\right)\right) J\left(F\left(u_{2}\right)\right) J\left(F\left(u_{3}\right)\right) \\
& \times m\left(u_{1}, u_{2}, u_{3}\right) d u_{1} d u_{2} d u_{3}, \\
& e_{2}=-\int_{-\infty}^{\infty} \int_{-\infty}^{\infty} \int_{-\infty}^{\infty} J\left(F\left(u_{1}\right)\right) J\left(F\left(u_{2}\right)\right) J^{(1)}\left(F\left(u_{3}\right)\right) \\
& \times k\left(u_{1}, u_{2}\right) k\left(u_{2}, u_{3}\right) d u_{1} d u_{2} d u_{3} .
\end{aligned}
$$


Then, from direct computations, we have

$$
\begin{aligned}
& E\left[g_{1}\left(X_{1}\right) \alpha\left(X_{1}\right)\right]=e_{1}+2 e_{2}, \quad E\left[v_{1}^{3}\left(X_{1}\right)\right]=\frac{e_{1}}{\sigma^{3}(J, F)}, \\
& E\left[v_{1}\left(X_{1}\right) v_{1}\left(X_{2}\right) v_{2}\left(X_{1}, X_{2}\right)\right]=-\frac{e_{1}}{\sigma^{3}(J, F)}-\frac{e_{2}}{\sigma^{3}(J, F)}, \\
& \tau=\frac{\eta}{\sigma(J, F)}-\frac{e_{1}+2 e_{2}}{2 \sigma^{3}(J, F)} \quad \text { and } \quad \kappa_{3}=-\frac{2 e_{1}+3 e_{2}}{\sigma^{3}(J, F)} .
\end{aligned}
$$

For $p$ and $q$ in the normalizing transformation, we have

$$
p=\frac{2 e_{1}+3 e_{2}}{6 \sigma^{3}(J, F)} \text { and } q=\frac{e_{1}+3 e_{2}}{6 \sigma^{3}(J, F)}-\frac{\eta}{\sigma(J, F)} .
$$

Substituting the empirical distribution $F_{n}(u)$ in the definitions of $\eta, e_{1}$ and $e_{2}$, we can get the following estimators $\hat{\eta}, \hat{e}_{1}$ and $\hat{e}_{2}$ :

$$
\begin{gathered}
\hat{\eta}=-\frac{1}{2} \int_{-\infty}^{\infty} J^{(1)}\left(F_{n}(u)\right) F_{n}(u)\left(1-F_{n}(u)\right) d u \\
\hat{k}(u, t)=F_{n}(\min (u, t))-F_{n}(u) F_{n}(t), \\
\hat{m}\left(u_{1}, u_{2}, u_{3}\right)=F_{n}\left(\min \left(u_{1}, u_{2}, u_{3}\right)\right)-F_{n}\left(u_{1}\right) F_{n}\left(\min \left(u_{2}, u_{3}\right)\right) \\
-F_{n}\left(u_{2}\right) F_{n}\left(\min \left(u_{1}, u_{3}\right)\right)-F_{n}\left(u_{3}\right) F_{n}\left(\min \left(u_{1}, u_{2}\right)\right) \\
+F_{n}\left(u_{1}\right) F_{n}\left(u_{2}\right) F_{n}\left(u_{3}\right), \\
\hat{e}_{1}=-\int_{-\infty}^{\infty} \int_{-\infty}^{\infty} \int_{-\infty}^{\infty} \begin{array}{c}
J\left(F_{n}\left(u_{1}\right)\right) J\left(F_{n}\left(u_{2}\right)\right) J\left(F_{n}\left(u_{3}\right)\right) \\
\times \hat{m}\left(u_{1}, u_{2}, u_{3}\right) d u_{1} d u_{2} d u_{3}, \\
\hat{e}_{2}=-\int_{-\infty}^{\infty} \int_{-\infty}^{\infty} \int_{-\infty}^{\infty} J\left(F_{n}\left(u_{1}\right)\right) J\left(F_{n}\left(u_{2}\right)\right) J^{(1)}\left(F_{n}\left(u_{3}\right)\right) \\
\times \hat{k}\left(u_{1}, u_{2}\right) \hat{k}\left(u_{2}, u_{3}\right) d u_{1} d u_{2} d u_{3} .
\end{array}
\end{gathered}
$$

Inverting the transformation $\pi(s)$ in (8), we have a confidence interval of $T(F)$ with confidence coefficient $1-\alpha$. The inversion is given by

$$
\pi^{-1}(t)=\frac{\sqrt{n}}{\hat{p}}\left\{1+\frac{3 \hat{p}}{\sqrt{n}}\left(t-\frac{\hat{q}}{\sqrt{n}}\right)\right\}^{1 / 3}-\frac{\sqrt{n}}{\hat{p}} .
$$

Then we have the following corollary.

Corollary. If the conditions of Theorem 4 and the equation (7) hold, we have

$$
\begin{aligned}
& P\left\{T\left(F_{n}\right)-\pi^{-1}\left(z_{\alpha / 2}\right) \frac{\hat{\sigma}(J, F)}{\sqrt{n}} \leq T(F) \leq T\left(F_{n}\right)+\pi^{-1}\left(z_{\alpha / 2}\right) \frac{\hat{\sigma}(J, F)}{\sqrt{n}}\right\} \\
= & 1-\alpha+o\left(n^{-1 / 2}\right)
\end{aligned}
$$

where $z_{\alpha / 2}$ is an upper $\alpha / 2$-point of the standard normal distribution. 
Then the above confidence interval has a second order accuracy. It is easy to show that for fixed $z_{\alpha / 2}$

$$
\pi^{-1}\left(z_{\alpha / 2}\right) \approx z_{\alpha / 2}-\frac{\hat{p} z_{\alpha / 2}^{2}+\hat{q}}{\sqrt{n}}
$$

\section{Examples.}

(1) For the score function $J(u)=1$, the corresponding $L$-statistic $T\left(F_{n}\right)$ is the sample mean and it is easy to check that the conditions for the score function in Theorem 4 and 5 are satisfied. From direct computation, we have

$$
\eta=0, \quad \sigma^{2}(J, F)=\operatorname{Var}\left(X_{1}\right), \quad e_{1}=E\left[\left\{X_{1}-E\left(X_{1}\right)\right\}^{3}\right] \quad \text { and } \quad e_{2}=0 .
$$

Thus we can apply Theorems 4 and 5 to the sample mean.

(2) We consider the score function $J(u)=4 u-2$ and the corresponding $T\left(F_{n}\right)$ is the Gini's mean difference. It is easy to check that the conditions for the score function in Theorem 4 and 5 are satisfied. Since

$$
\eta=-2 \int_{-\infty}^{\infty} F(u)\{1-F(u)\} d u,
$$

the bias $\eta$ is always negative. If the underlying distribution $F(u)$ is $N\left(\mu, \sigma^{2}\right)$, the bias $\eta=$ $-2 \sqrt{2} \sigma$. The asymptotic variance is

$$
\sigma^{2}(J, F)=8 \int_{-\infty}^{\infty} \int_{-\infty}^{t}\{2 F(t)-1\}\{1-F(t)\}\{2 F(u)-1\} F(u) d u d t .
$$

If the underlying distribution $F(u)$ is $N\left(\mu, \sigma^{2}\right)$, using the integration by parts, we can show that

$$
\sigma^{2}(J, F)=\sigma^{2}\left(\frac{16}{\pi}-\frac{40 \sqrt{3}}{3 \pi}+\frac{16}{\sqrt{\pi}}+\frac{4}{3}\right) .
$$

It may be possible to calculate $e_{1}$ and $e_{2}$, if the underlying distribution is normal. For constructing the confidence interval, we do not need to obtain explicit forms of $\sigma^{2}(J, F), \eta, e_{1}$ and $e_{2}$. We can use the estimators $\hat{\sigma}^{2}(J, F), \hat{\eta}, \hat{e}_{1}$ and $\hat{e}_{2}$ in (5), (9), (10) and (11).

\section{Proofs}

First we review the moment evaluations of the $H$-decomposition, which is very useful for discussing asymptotic properties of statistics. Let $v\left(x_{1}, \cdots, x_{r}\right)$ be a function which is symmetric in its arguments and $E\left[v\left(X_{1}, \cdots, X_{r}\right)\right]=0$. Let us define

$$
\begin{aligned}
\rho_{1}\left(x_{1}\right) & =E\left[v\left(x_{1}, X_{2}, \cdots, X_{r}\right)\right], \\
\rho_{2}\left(x_{1}, x_{2}\right) & =E\left[v\left(x_{1}, x_{2}, \cdots, X_{r}\right)\right]-\rho_{1}\left(x_{1}\right)-\rho_{1}\left(x_{2}\right), \cdots,
\end{aligned}
$$

and

$$
\rho_{r}\left(x_{1}, x_{2}, \cdots, x_{r}\right)=v\left(x_{1}, x_{2}, \cdots, x_{r}\right)-\sum_{k=1}^{r-1} \sum_{C_{r, k}} \rho_{k}\left(x_{i_{1}}, x_{i_{2}}, \cdots, x_{i_{k}}\right)
$$

where $\sum_{C_{n, k}}$ indicates that the summation is taken over all integers $i_{1}, \cdots, i_{k}$ satisfying $1 \leq i_{1}<$ $\cdots<i_{k} \leq n$. Then we can show that

$$
E\left[\rho_{k}\left(X_{1}, \cdots, X_{k}\right) \mid X_{1}, \cdots, X_{k-1}\right]=0 \text { a.s. }
$$


and

$$
\sum_{C_{n, r}} v\left(X_{i_{1}}, \cdots, X_{i_{r}}\right)=\sum_{k=1}^{r}\left(\begin{array}{l}
n-k \\
r-k
\end{array}\right) \Lambda_{k}
$$

where

$$
\Lambda_{k}=\sum_{C_{n, k}} \rho_{k}\left(X_{i_{1}}, \cdots, X_{i_{k}}\right) .
$$

Using the equation (12) and moment evaluations of martingales (Dharmadhikari, Fabian and Jogdeo (1968)), we have the upper bounds of the absolute moments of $\Lambda_{k}$ as follows.

Lemma 1. For $q \geq 2$, if $E\left|v\left(X_{1}, \cdots, X_{r}\right)\right|^{q}<\infty$, there exists a positive constant $C$, which may depend on $v$ and $F$ but not on $n$, such that

$$
E\left|\Lambda_{k}\right|^{q} \leq C n^{q k / 2} E\left|\rho_{k}\left(X_{i_{1}}, \cdots, X_{i_{k}}\right)\right|^{q} .
$$

Proof. See Fujioka and Maesono (2000).

Next we will obtain moment evaluations of $\left|F_{n}(u)-F(u)\right|$ and $\left|F_{n ; i}(u)-F_{n}(u)\right|$. From the definition we have

$$
F_{n ; i}(u)-F_{n}(u)=-\frac{1}{n-1} \tilde{I}\left(X_{i} ; u\right)+\frac{1}{n(n-1)} \sum_{j=1}^{n} \tilde{I}\left(X_{j} ; u\right) .
$$

Thus we have the following lemma.

Lemma 2. For $r \geq 2$, we have

$$
E\left|F_{n}(u)-F(u)\right|^{r} \leq C n^{-r / 2} F(u)(1-F(u))
$$

and

$$
E\left|F_{n ; i}(u)-F_{n}(u)\right|^{r} \leq C(n-1)^{-r} F(u)(1-F(u))
$$

where $C$ is a constant.

Proof. Since

$$
F_{n}(u)-F(u)=n^{-1} \sum_{i=1}^{n} \tilde{I}\left(X_{i} ; u\right)
$$

and $\left|\tilde{I}\left(X_{i} ; u\right)\right| \leq 1$, it follows from Lemma 1 that

$$
\begin{aligned}
E\left|F_{n}(u)-F(u)\right|^{r} & \leq C n^{-r / 2} E\left|\tilde{I}\left(X_{i} ; u\right)\right|^{r} \\
& \leq C n^{-r / 2} E\left[\tilde{I}\left(X_{i} ; u\right)\right]^{2} \\
& =C n^{-r / 2} F(u)(1-F(u)) .
\end{aligned}
$$

From the equation (15) and the Minkowski's inequality, we have

$$
\begin{aligned}
& {\left[E\left|F_{n ; i}(u)-F_{n}(u)\right|^{r}\right]^{1 / r} } \\
\leq & {\left[E\left|\frac{1}{n-1} \tilde{I}\left(X_{i} ; u\right)\right|^{r}\right]^{1 / r}+\left[E\left|\frac{1}{n(n-1)} \sum_{j=1}^{n} \tilde{I}\left(X_{j} ; u\right)\right|^{r}\right]^{1 / r} . }
\end{aligned}
$$


Since $\left|\tilde{I}\left(X_{i} ; u\right)\right| \leq 1$, we can show that

$$
\begin{aligned}
& E\left|\frac{1}{n-1} \tilde{I}\left(X_{i} ; u\right)\right|^{r}=(n-1)^{-r} E\left|\tilde{I}\left(X_{i} ; u\right)\right|^{r} \\
\leq & (n-1)^{-r} E\left|\tilde{I}\left(X_{i} ; u\right)\right|^{2}=(n-1)^{-r} F(u)(1-F(u)) .
\end{aligned}
$$

Further from Lemma 1, we can get

$$
\begin{aligned}
& E\left|\frac{1}{n(n-1)} \sum_{j=1}^{n} \tilde{I}\left(X_{j} ; u\right)\right|^{r}=C n^{-r}(n-1)^{-r} n^{r / 2} E\left|\tilde{I}\left(X_{1} ; u\right)\right|^{r} \\
\leq & C(n-1)^{-3 r / 2} F(u)(1-F(u)) .
\end{aligned}
$$

Thus we have the desired result.

Proof of Theorem 1. Let us define

$$
\begin{aligned}
& A_{1}^{(i)}=-\int_{-\infty}^{\infty} J\left(F_{n}(u)\right)\left[F_{n ; i}(u)-F_{n}(u)\right] d u, \\
& A_{2}^{(i)}=-\frac{1}{2} \int_{-\infty}^{\infty} J^{(1)}\left(F_{n}(u)\right)\left[F_{n ; i}(u)-F_{n}(u)\right]^{2} d u
\end{aligned}
$$

and

$$
R_{n}^{(i)}=T\left(F_{n ; i}\right)-T\left(F_{n}\right)-A_{1}^{(i)}-A_{2}^{(i)}
$$

Then we have

$$
\begin{gathered}
(n-1) \sum_{i=1}^{n}\left[T\left(F_{n ; i}\right)-T\left(F_{n}\right)\right]^{2} \\
=(n-1) \sum_{i=1}^{n}\left\{\left(A_{1}^{(i)}\right)^{2}+2 A_{1}^{(i)} A_{2}^{(i)}+\left(A_{2}^{(i)}\right)^{2}+\left(R_{n}^{(i)}\right)^{2}\right. \\
\left.\quad+2 A_{1}^{(i)} R_{n}^{(i)}+2 A_{2}^{(i)} R_{n}^{(i)}\right\} .
\end{gathered}
$$

We will study each term precisely.

$\left[(n-1) \sum_{i=1}^{n}\left(A_{1}^{(i)}\right)^{2}\right]$.

Hereafter, for the sake of simplicity, we use $\int$ instead of $\int_{-\infty}^{\infty}$. It follows from the equation (15) that

$$
\begin{aligned}
& (n-1) \sum_{i=1}^{n}\left(A_{1}^{(i)}\right)^{2} \\
= & \frac{1}{n-1} \sum_{i=1}^{n} \iint J\left(F_{n}(u)\right) J\left(F_{n}(t)\right) \tilde{I}\left(X_{i} ; u\right) \tilde{I}\left(X_{i} ; t\right) d u d t \\
& -\frac{1}{n(n-1)} \sum_{i=1}^{n} \sum_{j=1}^{n} \iint J\left(F_{n}(u)\right) J\left(F_{n}(t)\right) \tilde{I}\left(X_{i} ; u\right) \tilde{I}\left(X_{j} ; t\right) d u d t \\
= & \frac{1}{n} \sum_{i=1}^{n} \iint J\left(F_{n}(u)\right) J\left(F_{n}(t)\right) \tilde{I}\left(X_{i} ; u\right) \tilde{I}\left(X_{i} ; t\right) d u d t \\
& -\frac{2}{n(n-1)} \sum_{C_{n, 2}} \iint J\left(F_{n}(u)\right) J\left(F_{n}(t)\right) \tilde{I}\left(X_{i} ; u\right) \tilde{I}\left(X_{j} ; t\right) d u d t .
\end{aligned}
$$


Let us define

$$
\begin{aligned}
& a_{1}=\frac{1}{n} \sum_{i=1}^{n} \iint J(F(u)) J\left(F_{n}(t)\right) \tilde{I}\left(X_{i} ; u\right) \tilde{I}\left(X_{i} ; t\right) d u d t, \\
& a_{2}=\frac{1}{n} \sum_{i=1}^{n} \iint J^{(1)}(F(u)) J\left(F_{n}(t)\right)\left[F_{n}(u)-F(u)\right] \tilde{I}\left(X_{i} ; u\right) \tilde{I}\left(X_{i} ; t\right) d u d t, \\
& a_{3}=\frac{1}{n} \sum_{i=1}^{n} \iint J^{(2)}(F(u)) J\left(F_{n}(t)\right) \frac{1}{2}\left[F_{n}(u)-F(u)\right]^{2} \tilde{I}\left(X_{i} ; u\right) \tilde{I}\left(X_{i} ; t\right) d u d t
\end{aligned}
$$

and

$$
\begin{gathered}
a_{4}=\frac{1}{n} \sum_{i=1}^{n} \iint\left[J^{(2)}\left(F^{*}(u)\right)-J^{(2)}(F(u))\right] \\
\times\left(F_{n}(t)\right) \frac{1}{2}\left[F_{n}(u)-F(u)\right]^{2} \\
\times \tilde{I}\left(X_{i} ; u\right) \tilde{I}\left(X_{i} ; t\right) d u d t
\end{gathered}
$$

where $F_{n}^{*}(u)$ is between $F_{n}(u)$ and $F(u)$. Then expanding around $F(u)$, we have

$$
\begin{aligned}
& \frac{1}{n} \sum_{i=1}^{n} \iint J\left(F_{n}(u)\right) J\left(F_{n}(t)\right) \tilde{I}\left(X_{i} ; u\right) \tilde{I}\left(X_{i} ; t\right) d u d t \\
= & a_{1}+a_{2}+a_{3}+a_{4} .
\end{aligned}
$$

Further let us define

$$
\begin{aligned}
b_{1} & =\frac{1}{n} \sum_{i=1}^{n} \iint J(F(u)) J(F(t)) \tilde{I}\left(X_{i} ; u\right) \tilde{I}\left(X_{i} ; t\right) d u d t \\
b_{2} & =\frac{1}{n} \sum_{i=1}^{n} \iint J(F(u)) J^{(1)}(F(t))\left[F_{n}(t)-F(t)\right] \tilde{I}\left(X_{i} ; u\right) \tilde{I}\left(X_{i} ; t\right) d u d t, \\
b_{3} & =\frac{1}{n} \sum_{i=1}^{n} \iint J(F(u)) J^{(2)}(F(t)) \frac{1}{2}\left[F_{n}(t)-F(t)\right]^{2} \tilde{I}\left(X_{i} ; u\right) \tilde{I}\left(X_{i} ; t\right) d u d t
\end{aligned}
$$

and

$$
\begin{array}{r}
b_{4}=\frac{1}{n} \sum_{i=1}^{n} \iint J(F(u))\left[J^{(2)}\left(F_{n}^{* *}(t)\right)-J^{(2)}(F(t))\right] \frac{1}{2}\left[F_{n}(t)-F(t)\right]^{2} \\
\times \tilde{I}\left(X_{i} ; u\right) \tilde{I}\left(X_{i} ; t\right) d u d t
\end{array}
$$

where $F_{n}^{* *}(t)$ is between $F_{n}(t)$ and $F(t)$. Then expanding around $F(t)$, we have

$$
a_{1}=b_{1}+b_{2}+b_{3}+b_{4} \text {. }
$$

From the Lipshitz condition and boundedness of the score function $J(\cdot)$, we have

$$
E\left|b_{4}\right| \leq n^{-1} C \sum_{i=1}^{n} \iint E\left[\left|F_{n}(t)-F(t)\right|^{2+s}\left|\tilde{I}\left(X_{i} ; u\right) \tilde{I}\left(X_{i} ; t\right)\right| d u d t .\right.
$$

From the Hölder's inequality, we get

$$
\begin{aligned}
& E\left[\left|F_{n}(t)-F(t)\right|^{2+s}\left|\tilde{I}\left(X_{i} ; u\right) \tilde{I}\left(X_{i} ; t\right)\right|\right] \\
\leq & \left\{E\left[\left|F_{n}(t)-F(t)\right|^{4+2 s}\right]\right\}^{1 / 2}\left\{E\left|\tilde{I}\left(X_{i} ; u\right) \tilde{I}\left(X_{i} ; t\right)\right|^{2}\right\}^{1 / 2} .
\end{aligned}
$$


It follows from Lemma 2 that

$$
E\left[\left|F_{n}(t)-F(t)\right|^{4+2 s}\right] \leq C n^{-2-s} F(t)(1-F(t)) .
$$

Further we can show that for $u \leq t$

$$
\begin{aligned}
& E\left|\tilde{I}\left(X_{i} ; u\right) \tilde{I}\left(X_{i} ; t\right)\right|^{2} \\
= & F(u)(1-F(t))\{1-F(t)-2 F(u)+3 F(u) F(t)\} \\
\leq & 4 F(u)(1-F(t)) .
\end{aligned}
$$

Therefore, we get

$$
\begin{aligned}
& \iint\left\{E\left[\left|F_{n}(t)-F(t)\right|^{4+2 s}\right] E\left|\tilde{I}\left(X_{i} ; u\right) \tilde{I}\left(X_{i} ; t\right)\right|^{2}\right\}^{1 / 2} d u d t \\
\leq & C n^{-1-s / 2}\left[\iint_{u \leq t}\{F(t)(1-F(t)) F(u)(1-F(t))\}^{1 / 2} d u d t\right. \\
& \left.+\iint_{t \leq u}\{F(t)(1-F(t)) F(t)(1-F(u))\}^{1 / 2} d u d t\right] \\
\leq & C n^{-1-s / 2}\left[\iint_{u \leq t}\{F(t)(1-F(t)) F(u)(1-F(u))\}^{1 / 2} d u d t\right. \\
& \left.+\iint_{t \leq u}\{F(u)(1-F(t)) F(t)(1-F(u))\}^{1 / 2} d u d t\right] \\
= & C n^{-1-s / 2}\left[\int\{F(u)(1-F(u))\}^{1 / 2} d u\right]^{2} \\
= & O\left(n^{-1-s / 2}\right) .
\end{aligned}
$$

Thus we have $E\left|b_{4}\right|=O\left(n^{-1-s / 2}\right)=O\left(n^{-1 / 2-1 / 2-s / 2}\right)$ and $b_{4}=o_{L}\left(n^{-1 / 2}\right)$.

For $b_{1}$ we get

$$
\begin{aligned}
b_{1}= & \sigma^{2}(J, F) \\
& +n^{-1} \sum_{i=1}^{n} \iint J(F(u)) J(F(t))\left\{\tilde{I}\left(X_{i} ; u\right) \tilde{I}\left(X_{i}, t\right)-k(u, t)\right\} d u d t .
\end{aligned}
$$

For $b_{2}$, we have

$$
\begin{aligned}
b_{2}= & n^{-2} \sum_{i=1}^{n} \iint J(F(u)) J^{(1)}(F(t)) \tilde{I}\left(X_{i} ; u\right) \tilde{I}^{2}\left(X_{i} ; t\right) d u d t \\
& +n^{-2} \sum_{i=1}^{n} \sum_{j \neq i}^{n} \iint J(F(u)) J^{(1)}(F(t)) \tilde{I}\left(X_{i} ; u\right) \tilde{I}\left(X_{i} ; t\right) \tilde{I}\left(X_{j} ; t\right) d u d t \\
= & n^{-2} \sum_{i=1}^{n} Z_{i}+n^{-1} \iint J(F(u)) J^{(1)}(F(t))(1-2 F(t)) k(u, t) d u d t \\
& +n^{-2} \sum_{C_{n, 2}} \iint J(F(u)) J^{(1)}(F(t))\left\{\tilde{I}\left(X_{i} ; u\right) \tilde{I}\left(X_{i} ; t\right) \tilde{I}\left(X_{j} ; t\right)\right. \\
& \left.+\tilde{I}\left(X_{j} ; u\right) \tilde{I}\left(X_{j} ; t\right) \tilde{I}\left(X_{i} ; t\right)\right\} d u d t
\end{aligned}
$$


where

$$
\begin{aligned}
Z_{i}=\iint J(F(u)) J^{(1)}(F(t))\left\{\tilde{I}\left(X_{i} ; u\right) \tilde{I}^{2}\left(X_{i} ; t\right)\right. \\
\\
-(1-2 F(t)) k(u, t)\} d u d t
\end{aligned}
$$

It follows from the conditions of Theorem 1 that

$$
\begin{aligned}
E\left|Z_{i}\right|^{2} & \leq C \int \cdots \int E\left|\prod_{k=1}^{2} \tilde{I}\left(X_{i} ; u_{k}\right) \prod_{\ell=1}^{2} \tilde{I}^{2}\left(X_{i} ; t_{\ell}\right)\right| \prod_{k=1}^{2} d u_{k} \prod_{\ell=1}^{2} d t_{\ell} \\
& \leq C \int \cdots \int\left\{\prod_{k=1}^{2} E\left|\tilde{I}\left(X_{i} ; u_{k}\right)\right|^{4} \prod_{\ell=1}^{2} E\left|\tilde{I}\left(X_{i} ; t_{\ell}\right)\right|^{8}\right\}^{1 / 4} \prod_{k=1}^{2} d u_{k} \prod_{\ell=1}^{2} d t_{\ell} \\
& \leq C \prod_{k=1}^{2} \int\left\{F\left(u_{k}\right)\left(1-F\left(u_{k}\right)\right)\right\}^{1 / 4} d u_{k} \prod_{\ell=1}^{2} \int\left\{F\left(t_{\ell}\right)\left(1-F\left(t_{\ell}\right)\right)\right\}^{1 / 4} d t_{\ell} \\
& <\infty .
\end{aligned}
$$

Since $E\left(Z_{i}\right)=0$, we have

$$
E\left|n^{-2} \sum_{i=1}^{n} Z_{i}\right|^{2}=O\left(n^{-3}\right)=O\left(n^{-1 / 2-1-3 / 2}\right)
$$

and $n^{-2} \sum_{i=1}^{n} Z_{i}=o_{L}\left(n^{-1 / 2}\right)$. Here we get

$$
\begin{aligned}
& E\left[\tilde{I}\left(X_{1} ; u\right) \tilde{I}\left(X_{1} ; t\right) \tilde{I}\left(X_{2} ; t\right)+\tilde{I}\left(X_{2} ; u\right) \tilde{I}\left(X_{2} ; t\right) \tilde{I}\left(X_{1} ; t\right) \mid X_{1}=x\right] \\
= & k(u, t) \tilde{I}(x ; t) .
\end{aligned}
$$

Thus it follows from the $H$-decomposition that

$$
\begin{aligned}
& n^{-2} \sum_{C_{n, 2}} \iint J(F(u)) J^{(1)}(F(t)) \\
+\tilde{I}\left(X_{i} ; u\right) \tilde{I}\left(X_{i} ; t\right) \tilde{I}\left(X_{j} ; t\right) & \left.\tilde{I}\left(X_{j} ; t\right) \tilde{I}\left(X_{i} ; t\right)\right\} d u d t \\
= & n^{-1} \sum_{i=1}^{n} \iint J(F(u)) J^{(1)}(F(t)) k(u, t) \tilde{I}\left(X_{i} ; t\right) d u d t \\
+ & n^{-2} \sum_{C_{n, 2}} \iint J(F(u)) J^{(1)}(F(t)) k^{*}\left(X_{i}, X_{j} ; t ; u\right) d u d t
\end{aligned}
$$

where

$$
\begin{aligned}
& k^{*}\left(X_{i}, X_{j} ; t ; u\right) \\
= & \tilde{I}\left(X_{i} ; t\right) \tilde{I}\left(X_{j} ; t\right)\left\{\tilde{I}\left(X_{i} ; u\right)+\tilde{I}\left(X_{j} ; u\right)\right\}-k(u, t)\left\{\tilde{I}\left(X_{i} ; t\right)+\tilde{I}\left(X_{j} ; t\right)\right\} .
\end{aligned}
$$

Similarly we can show that

$$
\begin{aligned}
b_{3}= & \frac{1}{2 n} \iint J(F(u)) J^{(2)}(F(v)) k(u, v) F(v)(1-F(v)) d u d v \\
& +n^{-2} \sum_{C_{n, 2}} \iint J(F(u)) J^{(2)}(F(v)) k(u, v) \tilde{I}\left(X_{i} ; v\right) \tilde{I}\left(X_{j} ; v\right) d u d v \\
& +o_{L}\left(n^{-1 / 2}\right)
\end{aligned}
$$


and $b_{4}=o_{L}\left(n^{-1 / 2}\right)$.

For $a_{2}$, let us define

$$
c_{1}=n^{-2} \sum_{i=1}^{n} \sum_{j=1}^{n} \iint J^{(1)}(F(u)) J(F(v)) \tilde{I}\left(X_{i} ; u\right) \tilde{I}\left(X_{i} ; v\right) \tilde{I}\left(X_{j} ; u\right) d u d v
$$

and

$$
\begin{gathered}
c_{2}=n^{-3} \sum_{i=1}^{n} \sum_{j=1}^{n} \sum_{k=1}^{n} \iint J^{(1)}(F(u)) J^{(1)}(F(v)) \tilde{I}\left(X_{i} ; u\right) \tilde{I}\left(X_{i} ; v\right) \tilde{I}\left(X_{j} ; u\right) \\
\times \tilde{I}\left(X_{k} ; v\right) d u d v .
\end{gathered}
$$

Then, similarly as $a_{1}$, we have that $a_{2}=c_{1}+c_{2}+o_{L}\left(n^{-1 / 2}\right)$. Using $H$-decomposition, we can show that

$$
\begin{aligned}
c_{1}= & n^{-1} \iint J^{(1)}(F(u)) J(F(v)) k(u, v)\{1-2 F(u)\} d u d v \\
& +n^{-1} \sum_{i=1}^{n} \iint J^{(1)}(F(u)) J(F(v)) k(u, v) \tilde{I}\left(X_{i} ; u\right) d u d v \\
& +n^{-2} \sum_{C_{n, 2}} \iint J^{(1)}(F(u)) J(F(v)) k^{*}\left(X_{i}, X_{j} ; u ; v\right) d u d v \\
& +o_{L}\left(n^{-1 / 2}\right) .
\end{aligned}
$$

Similarly we have

$$
\begin{aligned}
c_{2}= & n^{-1} \iint J^{(1)}(F(u)) J^{(1)}(F(v)) k^{2}(u, v) d u d v \\
& +n^{-2} \sum_{C_{n, 2}} 2 \iint J^{(1)}(F(u)) J^{(1)}(F(v)) k(u, v) \tilde{I}\left(X_{i} ; u\right) \tilde{I}\left(X_{j} ; v\right) d u d v \\
& +o_{L}\left(n^{-1 / 2}\right) .
\end{aligned}
$$

Further for $a_{3}$, we can show that

$$
\begin{aligned}
a_{3}= & \frac{1}{2 n} \iint J^{(2)}(F(u)) J(F(v)) k(u, v) F(u)(1-F(u)) d u d v \\
& n^{-2} \sum_{C_{n, 2}} \iint J^{(2)}(F(u)) J(F(v)) k(u, v) \tilde{I}\left(X_{i} ; u\right) \tilde{I}\left(X_{j} ; u\right) d u d v \\
& +o_{L}\left(n^{-1 / 2}\right)
\end{aligned}
$$

It is easy to see that $a_{4}=o_{L}\left(n^{-1 / 2}\right)$ and, similarly to $b_{5}$, we can show that $a_{5}=o_{L}\left(n^{-1 / 2}\right)$. 
We will consider the second part of (19). Let us define

$$
\begin{gathered}
a_{1}^{*}=-\frac{2}{n(n-1)} \sum_{C_{n, 2}} \iint J(F(u)) J\left(F_{n}(v)\right) \tilde{I}\left(X_{i} ; u\right) \tilde{I}\left(X_{j} ; v\right) d u d v, \\
a_{2}^{*}=-\frac{2}{n(n-1)} \sum_{C_{n, 2}} \iint J^{(1)}(F(u)) J\left(F_{n}(v)\right)\left[F_{n}(u)-F(u)\right] \\
\times \tilde{I}\left(X_{i} ; u\right) \tilde{I}\left(X_{j} ; v\right) d u d v, \\
\begin{array}{r}
a_{3}^{*}=-\frac{2}{n(n-1)} \sum_{C_{n, 2}} \iint J^{(2)}(F(u)) J\left(F_{n}(v)\right) \frac{1}{2}\left[F_{n}(u)-F(u)\right]^{2} \\
\times \tilde{I}\left(X_{i} ; u\right) \tilde{I}\left(X_{j} ; v\right) d u d v, \\
a_{4}^{*}=-\frac{2}{n(n-1)} \sum_{C_{n, 2}} \iint J^{(3)}(F(u)) J\left(F_{n}(v)\right) \frac{1}{6}\left[F_{n}(u)-F(u)\right]^{3} \\
\times \tilde{I}\left(X_{i} ; u\right) \tilde{I}\left(X_{j} ; v\right) d u d v, \\
a_{5}^{*}=-\frac{2}{n(n-1)} \sum_{C_{n, 2}} \iint\left[J^{(3)}\left(F_{n}^{*}(u)\right)-J^{(3)}(F(u))\right] J\left(F_{n}(v)\right) \\
\times \frac{1}{6}\left[F_{n}(u)-F(u)\right]^{3} \tilde{I}\left(X_{i} ; u\right) \tilde{I}\left(X_{j} ; v\right) d u d v
\end{array}
\end{gathered}
$$

where $F_{n}^{*}(u)$ is between $F_{n}(u)$ and $F(u)$. Then expanding around $F(u)$, we have

$$
\begin{aligned}
& -\frac{2}{n(n-1)} \sum_{C_{n, 2}} \iint J\left(F_{n}(u)\right) J\left(F_{n}(v)\right) \tilde{I}\left(X_{i} ; u\right) \tilde{I}\left(X_{j} ; v\right) d u d v \\
= & a_{1}^{*}+a_{2}^{*}+a_{3}^{*}+a_{4}^{*}+a_{5}^{*} .
\end{aligned}
$$

Similarly as $a_{1}$, we can show that

$$
a_{1}^{*}=-n^{-2} \sum_{C_{n, 2}} 2 \iint J(F(u)) J(F(v)) \tilde{I}\left(X_{i} ; u\right) \tilde{I}\left(X_{j} ; v\right) d u d v+o_{L}\left(n^{-1 / 2}\right) .
$$

Further we can show that $a_{2}^{*}, a_{3}^{*}, a_{4}^{*}$ and $a_{5}^{*}$ are all $o_{L}\left(n^{-1 / 2}\right)$.

$\left[(n-1) \sum_{i=1}^{n} 2 A_{1}^{(i)} A_{2}^{(i)}\right]$

It follows from $H$-decomposition and Lemma 2 that

$$
\begin{aligned}
& (n-1) \sum_{i=1}^{n} 2 A_{1}^{(i)} A_{2}^{(i)} \\
= & (n-1) \sum_{i=1}^{n} \iint J\left(F_{n}(u)\right) J^{(1)}\left(F_{n}(v)\right)\left[F_{n ; i}(u)-F_{n}(u)\right] \\
& \times\left[F_{n ; i}(v)-F_{n}(v)\right]^{2} d u d v \\
= & -(n-1)^{-2} \sum_{i=1}^{n} \iint J\left(F_{n}(u)\right) J^{(1)}\left(F_{n}(v)\right) \tilde{I}\left(X_{i} ; u\right) \tilde{I}^{2}\left(X_{j} ; v\right) d u d v \\
& +o_{L}\left(n^{-1 / 2}\right) .
\end{aligned}
$$


Similarly to $a_{1}$, we can show that

$$
\begin{aligned}
& (n-1) \sum_{i=1}^{n} 2 A_{1}^{(i)} A_{2}^{(i)} \\
= & -n^{-1} \iint J(F(u)) J^{(1)}(F(v)) k(u, v)(1-2 F(v)) d u d v+o_{L}\left(n^{-1 / 2}\right) .
\end{aligned}
$$

For the rest terms of (18), we can show them $o_{L}\left(n^{-1 / 2}\right)$. Here we consider $(n-1) \sum_{i=1}^{n} 2 A_{1}^{(i)} R_{n}^{(i)}$. Here we have

$$
\begin{aligned}
E\left|(n-1) \sum_{i=1}^{n} 2 A_{1}^{(i)} R_{n}^{(i)}\right| & \leq 2 n(n-1) E\left|A^{(1)} R_{n}^{(1)}\right| \\
& \leq 2 n(n-1)\left\{E\left(A_{1}^{(1)}\right)^{2} E\left(R_{n}^{(1)}\right)^{2}\right\}^{1 / 2} .
\end{aligned}
$$

From Lemma 2, we can show that

$$
\begin{aligned}
& E\left(A_{1}^{(1)}\right)^{2} \\
= & \iint E\left\{J\left(F_{n}(u)\right) J\left(F_{n}(v)\right)\left[F_{n ; 1}(u)-F_{n}(u)\right]\left[F_{n ; 1}(v)-F_{n}(v)\right]\right\} d u d v \\
\leq & C \iint\left\{E\left[F_{n ; 1}(u)-F_{n}(u)\right]^{2} E\left[F_{n ; 1}(v)-F_{n}(v)\right]^{2}\right\}^{1 / 2} d u d v \\
\leq & C(n-1)^{-2} \iint\{F(u)(1-F(u))\}^{1 / 2}\{F(v)(1-F(v))\}^{1 / 2} d u d v \\
= & O\left(n^{-2}\right) .
\end{aligned}
$$

Similarly, it follows from the Lipshitz condition that

$$
\begin{aligned}
& E\left(R_{n}^{(1)}\right)^{2} \\
\leq & C \iint E\left\{\left|F_{n ; 1}(u)-F_{n}(u)\right|^{3+s}\left|F_{n ; 1}(v)-F_{n}(v)\right|^{3+s}\right\} d u d v \\
\leq & C \iint\left\{E\left[\left|F_{n ; 1}(u)-F_{n}(u)\right|^{6+2 s}\right] E\left[\left|F_{n ; 1}(v)-F_{n}(v)\right|^{6+2 s}\right]\right\}^{1 / 2} d u d v \\
= & O\left(n^{-6-2 s}\right) .
\end{aligned}
$$

Thus we have

$$
E\left|(n-1) \sum_{i=1}^{n} 2 A_{1}^{(i)} R_{n}^{(i)}\right|=O\left(n^{-2-s}\right)
$$

and so $(n-1) \sum_{i=1}^{n} 2 A_{1}^{(i)} R_{n}^{(i)}=o_{L}\left(n^{-1 / 2}\right)$.

From (20) (27), we have Theorem 1.

Proof of Theorem 2. Let us define

$$
\begin{aligned}
& d_{1}=-\int J(F(u))\left[F_{n}(u)-F(u)\right] d u, \\
& d_{2}=-\frac{1}{2} \int J^{(1)}(F(u))\left[F_{n}(u)-F(u)\right]^{2} d u, \\
& d_{3}=-\frac{1}{6} \int J^{(2)}(F(u))\left[F_{n}(u)-F(u)\right]^{3} d u
\end{aligned}
$$


and

$$
d_{4}=-\frac{1}{24} \int J^{(3)}(F(u))\left[F_{n}(u)-F(u)\right]^{4} d u .
$$

Under the conditions of Theorem 1, it follows from Lemma 2 that

$$
\begin{aligned}
& E\left|\sqrt{n}\left\{T\left(F_{n}\right)-T(F)\right\}-\sum_{i=1}^{4} d_{i}\right|^{2} \\
\leq & C \iint E\left[\left|F_{n}(u)-F(u)\right|^{4+s}\left|F_{n}(v)-F(v)\right|^{4+s} d u d v\right. \\
= & O\left(n^{-3-s}\right)
\end{aligned}
$$

and then

$$
\sqrt{n}\left\{T\left(F_{n}\right)-T(F)\right\}=\sqrt{n} \sum_{i=1}^{4} d_{i}+o_{L}\left(n^{-1 / 2}\right) .
$$

It is easy to show that

$$
\begin{aligned}
d_{1}= & -\frac{1}{n} \sum_{i=1}^{n} \int J(F(u)) \tilde{I}\left(X_{i} ; u\right) d u \\
d_{2}= & -\frac{1}{2 n} \int J^{(1)}(F(u)) F(u)(1-F(u)) d u \\
& -\frac{1}{2 n^{2}} \sum_{i=1}^{n} \int J^{(1)}(F(u))\left\{\tilde{I}^{2}\left(X_{i} ; u\right)-F(u)(1-F(u))\right\} d u \\
& -n^{-2} \sum_{C_{n, 2}} \int J^{(1)}(F(u)) \tilde{I}\left(X_{i} ; u\right) \tilde{I}\left(X_{j} ; u\right) d u, \\
d_{3}= & -\frac{1}{2 n^{2}} \sum_{i=1}^{n} \int J^{(2)} F(u)(1-F(u)) \tilde{I}\left(X_{i} ; u\right) d u \\
& -n^{-3} \sum_{C_{n, 3}} \int J^{(2)}(F(u)) \tilde{I}\left(X_{i} ; u\right) \tilde{I}\left(X_{j} ; u\right) \tilde{I}\left(X_{k} ; u\right) d u \\
& +n^{-1 / 2} o_{L}\left(n^{-1 / 2}\right)
\end{aligned}
$$

and $d_{4}=n^{-1 / 2} o_{L}\left(n^{-1 / 2}\right)$. Thus we have the equation (4).

Using Taylor expansion, we have

$$
\hat{\sigma}=\sigma+\frac{1}{2 \sigma}\left(\hat{\sigma}^{2}-\sigma^{2}\right)-\frac{1}{8 \sigma^{3}}\left(\hat{\sigma}^{2}-\sigma^{2}\right)^{2}+\frac{1}{16\left(\sigma^{*}\right)^{5}}\left(\hat{\sigma}^{2}-\sigma^{2}\right)^{3}
$$

where $\left(\sigma^{*}\right)^{2}$ is between $\sigma^{2}$ and $\hat{\sigma}^{2}$. Let us define

$$
D_{1}=\sum_{i=1}^{n} \alpha_{1}\left(X_{i}\right) \quad \text { and } \quad D_{2}=\sum_{C_{n, 2}} \alpha_{2}\left(X_{i}, X_{j}\right)
$$

Then we have

$$
\hat{\sigma}^{2}-\sigma^{2}=n^{-1} \delta+n^{-1} D_{1}+n^{-2} D_{2}+o_{L}\left(n^{-1 / 2}\right) .
$$


Using Lemma 1, it is easy to see that

$$
n^{-2} D_{1}^{2}=n^{-1} E\left[\alpha_{1}^{2}\left(X_{1}\right)\right]+n^{-2} \sum_{C_{n, 2}} 2 \alpha_{1}\left(X_{i}\right) \alpha_{2}\left(X_{j}\right)+o_{L}\left(n^{-1 / 2}\right) .
$$

Similarly we can show that

$$
\begin{aligned}
E\left|n^{-3} D_{1} D_{2}\right|^{2+\varepsilon / 2} & \leq n^{-6-3 \varepsilon}\left\{E\left|D_{1}\right|^{4+\varepsilon} E\left|D_{2}\right|^{4+\varepsilon}\right\}^{1 / 2} \\
& =O\left(n^{-3-3 \varepsilon / 4}\right)
\end{aligned}
$$

and then $n^{-3} D_{1} D_{2}=o_{L}\left(n^{-1 / 2}\right)$. We also have $n^{-4} D_{2}^{2}=o_{L}\left(n^{-1 / 2}\right)$. Thus we have

$$
\left(\hat{\sigma}^{2}-\sigma^{2}\right)^{2}=n^{-1} E\left[\alpha_{1}^{2}\left(X_{1}\right)\right]+n^{-2} \sum_{C_{n, 2}} 2 \alpha_{1}\left(X_{i}\right) \alpha_{1}\left(X_{j}\right)+o_{L}\left(n^{-1 / 2}\right) .
$$

Since $\sigma>0$, we have

$$
\begin{gathered}
P\left\{\left|\frac{1}{16\left(\sigma^{*}\right)^{5}}\left(\hat{\sigma}^{2}-\sigma^{2}\right)^{3}\right| \geq n^{-1}(\log n)^{-1}\right\} \\
\leq P\left\{\left|\sigma^{*}\right| \leq \frac{1}{2} \sigma\right\}+P\left\{\frac{2}{\sigma^{5}}\left|\hat{\sigma}^{2}-\sigma^{2}\right|^{3} \geq n^{-1}(\log n)^{-1}\right\} .
\end{gathered}
$$

It follows from $\left|\left(\sigma^{*}\right)^{2}-\sigma^{2}\right| \leq\left|\hat{\sigma}^{2}-\sigma^{2}\right|$ that

$$
\begin{gathered}
P\left\{\left|\left(\sigma^{*}\right)^{2}-\sigma^{2}\right| \geq \frac{1}{2} \sigma^{2}\right\} \\
\leq P\left\{\left|\hat{\sigma}^{2}-\sigma^{2}\right| \geq \frac{1}{2} \sigma^{2}\right\}=o\left(n^{-1 / 2}\right) .
\end{gathered}
$$

If we can show that $\left(\hat{\sigma}^{2}-\sigma^{2}\right)^{3}=o_{L}\left(n^{-1 / 2}\right)$, we have the equation (5). It is easy to see that

$$
\begin{aligned}
& P\left\{\left|n^{-1} D_{1} o_{L}\left(n^{-1 / 2}\right)\right| \geq n^{-1}(\log n)^{-1}\right\} \\
\leq & P\left\{\left|n^{-1} D_{1}\right| \geq 1\right\}+P\left\{\left|o_{L}\left(n^{-1 / 2}\right)\right| \geq n^{-1}(\log n)^{-1}\right\} \\
= & o\left(n^{-1 / 2}\right) .
\end{aligned}
$$

Similarly we get $n^{-2} D_{2} o_{L}\left(n^{-1 / 2}\right)=o_{L}\left(n^{-1 / 2}\right)$. Further applying $H$-decomposition, we can show that

$$
n^{-1} D_{1} n^{-2} \sum_{C_{n, 2}} 2 \alpha_{1}\left(X_{i}\right) \alpha_{1}\left(X_{j}\right)=o_{L}\left(n^{-1 / 2}\right)
$$

and

$$
n^{-2} D_{2} n^{-2} \sum_{C_{n, 2}} 2 \alpha_{1}\left(X_{i}\right) \alpha_{1}\left(X_{j}\right)=o_{L}\left(n^{-1 / 2}\right) .
$$

It is easy to see that $n^{-2} E\left[\alpha_{1}^{2}\left(X_{1}\right)\right] D_{1}=o_{L}\left(n^{-1 / 2}\right)$ and $n^{-3} E\left[\alpha_{1}^{2}\left(X_{1}\right)\right] D_{2}=o_{L}\left(n^{-1 / 2}\right)$. Therefore we have $\left(\hat{\sigma}^{2}-\sigma^{2}\right)^{3}=o_{L}\left(n^{-1 / 2}\right)$. This completes the proof of Theorem 2 .

Proof of Theorem 3. Since the studentized $L$-statistic is a special case of the ratio statistics and for the ratio statistic, Maesono (2003) has obtained the stochastic approximation with remainder term $o_{L}\left(n^{-1 / 2}\right)$. Then applying the result of Maesono (2003), we can obtain Theorem 3. 
Proof of Theorem 4. From Lai and Wang (1993), we have

$$
\begin{aligned}
& P\left\{\frac{\sqrt{n}\left\{T\left(F_{n}\right)-T(F)\right\}}{\hat{\sigma}(J, F)} \leq x\right\} \\
= & P\left\{\frac{\sqrt{n}\left\{T\left(F_{n}\right)-T(F)\right\}}{\hat{\sigma}(J, F)}-n^{-1 / 2} \tau \leq x-n^{-1 / 2} \tau\right\} \\
= & \Phi\left(x-n^{-1 / 2} \tau\right)-n^{-1 / 2} \phi\left(x-n^{-1 / 2} \tau\right) P_{1}\left(x-n^{-1 / 2} \tau\right) \\
& -n^{-1} \phi\left(x-n^{-1 / 2} \tau\right) P_{2}\left(x-n^{-1 / 2} \tau\right)+o\left(n^{-1 / 2}\right) .
\end{aligned}
$$

Using Taylor expansion, we can easily obtain the Edgeworth expansion of the studentized $L$ statistic.

\section{Acknowledgement}

This research was supported by Grant-in-Aid for Scientific Research of the Ministry of Education, Science and Culture under Contract Number 16340026(B), Japan.

\section{References}

Alberink, I. V., Pap, G. and van Zuijlen, M. C. A. (2001). Edgworth expansions for $L$-statistics, Prob. Math. Statist. 21, 277-302.

Boos, D. D. and Serfling, R. J. (1979). On Berry-Esséen rates for statistical functions with application to L-estimates. Report No. M499, Florida State Univ.

Dharmadhikari, S. W., Fabian, V. and Jogdeo, K. (1968). Bounds on the moments of martingales. Ann. Math. Statist. 39, 1719-1723.

Fujioka, Y. and Maesono, Y. (2000). Higher order normalizing transformations for asymptotic U-statistics. Jour. Statist. Plan. \& Inf. 83, 47-74.

Hall, P. (1992). On the removal of skewness by transformation. Jour. Royal. Statist. Soc. B 54, 221-228.

Helmers, R. (1982). Edgeworth expansions for linear combinations of order statistics. Math. Centre Tracts 105, Amsterdam.

Hoeffding, W. (1961). The strong law of large numbers for $U$-statistics. Univ. of North Carolina Institute of statistics. Mimeo Series. No.302.

Konishi, S. (1981). Normalizing transformations of some statistics in multivariate analysis. Biometrika, 68, 647-651.

Lai, T. L. and Wang, J. Q. (1993). Edgeworth expansion for symmetric statistics with applications to bootstrap methods. Statistica Sinica 3, 517-542.

Maesono, Y. (2003). Mean squared errors of ratio statistics and bias corrections. CMA Statistics Research Reports, The Australian National University, No. SRR 03-001.

Parr, W. C. and Schucany, W. R. (1982). Jackknifing $L$-statistics with smooth weight functions. Jour. Amer. Statist. Assoc. 77, 629-638. 
Petrov, V. V. (1995). Limit Theorems of Probability Theory: Sequences of Independent Random Variables. Oxford Sci. Publ., New York.

Serfling, R. J. (1980). Approximation Theorems of Mathematical Statistics. Wiley, New York.

Serfling, R. J. (1984). Generalized L-, $M$ - and $R$-statistics. Ann. Statist. 12, 76-86.

Shao, J. (1991). Jackknife variance estimators for generalized L-statistics. Statist. Prob. Lett. 11, 27-32.

Received June 26, 2006

Revised September 26, 2007 